Rev. Elev. Méd. vét. Pays' trop., 1975, 28 (1) : 17-19

\title{
Isolement de Salmonella Nairobi d'un zébu Indu-Brasil en République du Zaïre
}

\author{
par F. BERTELE (*) et J. P. ADRIANSENS (**)
}

\begin{abstract}
RESUME
Une entérobactérie ayant les caractéristiques d'une salmonelle est isolée en culture pure des prélèvements provenant d'un taurillon de race Indu-Brasil, mort quinze jours après le déclenchement d'une entérite aiguë. L'identification révèle qu'il s'agit de Salmonella nairobi. La seule salmonelle isolée jusque là dans cette région était $S$. dublin.

Il s'agit du premier isolement de $S$. nairobi au Zaïre et du second isolement en Afrique à partir de l'espèce bovine.
\end{abstract}

Dans la région de l'Ubangi, en République du Zaïre, sur des plateaux couverts d'une savane guinéenne à base d'Imperata cylindrica et Hyparrhenia diplandra, à $600 \mathrm{~m}$ d'altitude, se trouvent trois élevages dont le cheptel bovin dépasse 30.000 têtes.

La pathologie y est dominée par les trypanosomoses et des entérotoxémies d'étiologie non élucidée.

Les galeries forestières sont infestées par les glossines appartenant aux groupes: $G$. palpalis, G. morsitans, G. fusca. L'interdiction de la chasse dans les élevages y a préservé le gibier. Buffles, antilopes, phacochères, et éléphants sont nombreux.

Les tiques, abondantes sur le gibier, sont bien contrôlées dans les élevages par un dipping hebdomadaire.

Depuis 15 ans, à l'époque de l'année où les pluies sont les plus abondantes, on remarque

(*) F. Bertele, Dr. Vét., Compagnic Africaine d'Elevages, Lombo, B.P. 69 Libenge (République du Zaïre)

(**) J. P. Adriansens, Ir. Agr., Coopération Technique Belge, Ferme d'état de la Lola, B.P. 153 Gemena (République du Zaïre). parmi le bétail des cas d'entérite aiguë dont la manifestation la plus évidente est une diarrhée violente, d'odeur fétide, contenant des caillots de sang et de membranes catarthales. La morbidité est normalement peu élevée (10 p. 100). Le taux de mortalité varie de 20 à 30 p. 100 des bêtes malades.

En 1959, le laboratoire vétérinaire de Lubumbashi isola Salmonella dublin des prélèvements provenant du ranch de la Lombo. Depuis lors $S$. dublin est présumée responsable des cas de maladie qui se manifestent par des diarrhées violentes avec sang et catarrhe intestinal, comme indiqué plus haut.

Le traitement à base de sulfamides et de chloramphénicol, s'il est employé au début de la maladie, donne des résultats.

A partir de 1960 , en raison des difficultés de communication avec Lubumbashi, les prélèvements furent envoyés en Belgique et plus rarement au laboratoire de l'I.E.M.V.T. à Farcha. Les résultats furent toujours négatifs, sans doute par suite du mauvais état dans lequel les prélèvements arrivaient aux laboratoires. Il fut alors décidé d'équiper sur place un petit laboratoire avec des moyens très simples, mais permettant d'effectuer quelques re- 
cherches bactériologiques; les bactéries une fois isolées auraient été expédiées à des laboratoires spécialisés pour identification complète. En 1969, lors d'une épidémie grave, le laboratoire de l'Institut National de Recherches Vétérinaires d'Uccle (Belgique) isola de nouveau $S$. dublin d'un prélèvement arrivé en bonnes conditions. Le même résultat fut obtenu par le laboratoire local qui s'occupa du diagnostic de la maladie de 1969 à 1972, suite au passage de l'épidémie d'un ranch à l'autre.

Les identifications furent toujours confirmées par le laboratoire d'Uccle et parfois par l'Istituto Zooprofilattico delle Venezie à $\mathrm{Pa}$ doue (Italie) comme étant $S$. dublin.

En juin 1973, on introduisit au Zaïre, à l'occasion de la foire internationale de Kinshasa, du bétail brésilien. En octobre, après un séjour au domaine présidentiel de la Nsele où du bétail laitier hollandais (Pie Noire) se trouve depuis 1971 sans manifester des signes spéciaux de maladie, la grosse partie des taurillons et génisses brésiliennes fut transférée dans un des trois ranches de l'Ubangi, la Ferme d'Etat de la Lola.

Le 23 janvier 1974, un taurillon d'environ trente mois manifeste de la diarrhée violente; il est traité pendant quatre jours avec du chloramphénicol, la diarrhée s'arrête au quatrième jour, mais la bête reste en anorexie. La mort survient le 5 février.

Signalons qu'une génisse de même race tombe malade en même temps que le taurillon, mais elle guérit après avoir reçu le même traitement. Les examens, effectués pendant que le taurillon était encore vivant, avaient permis d'exclure comme maladie secondaire la trypanosomiase et la verminose. Une heure après la mort sont prélevés et envoyés au laboratoire local : un morceau de foie et de rate, un ganglion mésentérique et le ganglion précrưral. Un os long est envoyé enrobé de paraffine au laboratoire de l'I.E.M.V.T. à Farcha, N'djaména (Tchad).

Le foie, la rate et les deux ganglions sont ensemencés dans du bouillon au sélénite de sodium (Difco 0275-02). Le tube contenant le prélèvement du foie reste inexplicablement stérile, tandis que les trois autres donnent une flore bactérienne bien évidente.
Du bouillon au sélénite, les cultures sont passées sur un milieu au désoxycholate citrate - lactose àgar (Difco 0759-02) où elles se développent en colonies ayant les caractéristiques d'une salmonelle à l'état pur. Du désoxycholate, on passe sur le milieu de Kligler (Difco 0086-02). Vingt-quatre heures après l'ensemencement on remarque : présence de gaz; $\mathrm{H}_{2} \mathrm{~S}+$; culot jaune et pente rouge.

Une séro-agglutination rapide sur lame est effectuée avec un sérum (Behringwerke) polyvalent antisalmonella $O$, pour les groupes $A$. B. C. D. E. L'agglutination a lieu mais d'une façon très lente et avec des granulations très petites, différentes des granulations obtenues avec une souche de $S$. dublin employée comme témoin pour contrôler l'efficacité du sérum.

La souche conservée sur gélose est confiée au laboratoire de Farcha qui confirma qu'il s'agissait d'une salmonelle: Salmonella nairobi du groupe $\mathrm{T}$, formule antigénique : 42 ,r. Le laboratoire de l'I.E.M.V.T. à Farcha a isolé, à partir de la moelle osseuse de l'os long envoyé, la même entérobactérie en culture pure.

L'existence de l'agglutination en petits flocons s'explique aisément par la présence de co-agglutinines de l'antigène 42 dans le sérum polyvalent utilisé pour le diagnostic.

\section{CONCLUSION}

L'apparition de deux cas d'entérite aiguë, avec les caractéristiques d'une diarrhée salmonellique en pleine saison sèche, n'entre pas dans le schéma pathologique connu dans les élevages de l'Ubangi.

La présence de $S$. nairobi chez un taurillon importé soulève le problème de l'origine de cette bactérie.

Il est possible que la bactérie qui a provoqué la mort du zébu ait été importée du Brésil, mais l'hypothèse d'une souche locale paraît plus vraisemblable. Des investigations ultérieures préciseront nos connaissances à ce sujet. Dans ce contexte, il est utile de rappeler que $S$. nairobi, dont la souche Zaïroise représente la $4^{e}$ souche mondiale (2), a été isolée par HUMMEL à Dar-es-Salaam à partir d'un bovin d'abattoir apparemment en bonne santé (1). 


\section{SUMMARY}

Isolation of Salmonella nairobi from an Indu-Brazil zebu in Zaire

An enterobacteria with the characteristics of a salmonella is isolated in pure culture from material taken from an Indu-Brazil bull calf dead 15 days after the beginning of an acute enteritis. It was identified as Salmonella nairobi. Until then $S$. dublin alone was isolated in this area.

This is the first isolation of $S$. nairobi in Zaire and the second isolation from cattle in Africa.

\section{RESUMEN}

\section{Aislamiento de Salmonella nairobi de un cebú Indu-Brasil en República de Zaïre}

Se aisló en cultivo puro una enterobacteria, teniendo las características de una salmonela, a partir de muestras de un novillo de raza Indu-Brasil, muerto quince días después del principio de una enteritis aguda. Según la identificación, se trata de Salmonella nairobi. La sola salmonela aislada hasta ahora en esta región era $S$. dublin.

Es el primer aislamiento de $S$. nairobi en Zaïre y el segundo en Africa a partir de la especie bovina.

\section{BIBLIOGRAPHIE}

1. HUMMEL (P. H.). Isolation of Salmonella from domestic animals in Tanzania. Bull. epiz. Dis. Afr., 1969, $17: 305-310$.
2. KELTERBORN (E.). Salmonella species. La Haye, N.V. Junk Publ., 1967, pp. 596 - D 2.W. Et communication personnelle. 